\title{
65824
}

\section{MATRIX TRANSFORMATIONS FOR SPACECRAFT ATTITUDE DETERMINATION}

\section{DAVID P. CAUFFMAN}

GSFC

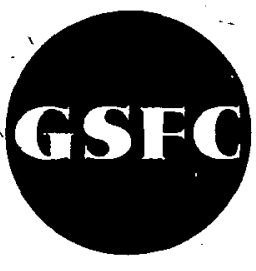

I

(NASA-TH-X-65824)

FOR SPACECRAFT ATTITUDE DETERMINATION D. P.
Cauffman (NASA) Jan. $1972 \mathrm{~g}$ p CSCL $12 \mathrm{~A}$
JANUARY 1972

Reproduced by

NATIONAL TECHNICAL

INFORMATION SERVICE U S Department of Commerco
Springfield VA 22151

GODDARD SPACE FLIGHT CENTER

GREENBELT, MARYLAND

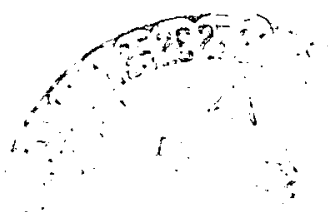


Matrix Transformations for Spacecraft Attitude Determination

\author{
by \\ David P. Cauffman* \\ Code 645, Goddard Space Flight Center \\ Greenbe1t, Maryland 20771
}

January, 1972

*National Academy of Sciences

Research Associate, NASA-Goddard Space Flight Center 


\section{Abstract}

A common problem for experimental space physicists is the determination of the "attitude matrix" $T$ which transforms vectors between representations in $X$ and $X^{\prime}$ coordinate systems according to $\vec{V}_{X}=T_{X X}, \vec{V}_{X^{\prime}}$. Solution of this problem using the standard Eulerian angle techniques is in general tedious and circuitous. A straightforward, simple, and efficient solution for the transformation matrix is a "double-cross" transformation, such as

$$
\mathrm{T}_{\mathrm{XX}} \mathrm{X}^{\prime}=[\hat{\mathrm{A}}, \mathrm{A} \hat{\mathrm{x}} \mathrm{B}, \hat{\mathrm{A}} \mathrm{x}(\mathrm{A} \hat{\mathrm{x}} \mathrm{B})]_{\mathrm{X}}[\hat{\mathrm{A}}, \mathrm{A} \hat{\mathrm{x}} \mathrm{B}, \hat{\mathrm{A}} \mathrm{x}(\mathrm{A} \hat{\mathrm{x}} \mathrm{B})]_{\mathrm{X}^{\prime}}^{\mathrm{t}} \text {, }
$$

which is calculated from any two directions $\hat{A}$ and $\hat{B}$ which are known in both $X$ and $X^{\prime}$ coordinates. The $\hat{B}$ direction need be known only well enough to define the plane in which $\hat{A}$ and $\hat{B}$ lie. The problem of the intersection of two cones as applicable to attitude solutions is also discussed. 


\section{Introduction}

Experimental space physicists frequently encounter the problem of determining the attitude of their sensors on rockets or satellites. The purpose of this report is to present a simple, efficient approach to the mathematical aspects of the problem of vehicle and sensor orientation.

It is well known that a (column) vector $\vec{V}_{X^{\prime}}$ defined in an orthogonal coordinate system $X^{\prime}$ may be transformed to another orthogonal coordinate system $X$ having the same origin by a transformation of the form

$$
\overrightarrow{\mathrm{v}}_{\mathrm{X}}=\mathrm{T}_{\mathrm{XX}} \cdot \overrightarrow{\mathrm{V}}_{\mathrm{X}}
$$

where $T$ is an orthonormal rotation, or attitude, matrix. Due to the orthonormal property of $T$, the inverse transformation is performed by the transpose of $\mathrm{T}$ :

$$
\overrightarrow{\mathrm{V}}_{\mathrm{X}}=\mathrm{T}_{\mathrm{XX}}{ }^{\prime} \mathrm{V}_{\mathrm{X}}
$$

The problem we shall address is to determine $T(\hat{A}, \hat{B})$ when any two directions, $\hat{A}$ and $\hat{B}$, are known in both $X$ and $X^{\prime}$ representations. (The mathematics are, of course, equally applicable in terms of the alternative physical interpretation, that the transformation rotates $\hat{A}$ and $\hat{B}$ to new positions in a fixed coordinate system.)

The usual method of expressing $T$ involves Eulerian angles. (See, for example, Mechanics texts such as Goldstein [1950], Marion [1965], and others, or app1ications-oriented volumes such as Thomson [1961], Singer [1964] or Greensite [1970].) The approach using Eulerian angles is suitable for physical problems involving rotations through known angles; however, it is difficult to apply in the situation being considered, and is cumbersome and expensive for computer applications due to the numerous 
trigonometric functions involved (29 factors which are either the sine or cosine of one of three angles). We shall derive an expression for $T(\hat{A}, \hat{B})$ which involves no trigonometric functions.

II. The Double-Cross Transformation

If a matrix $M$ composed of three (or more) column vectors replaces $\vec{V}$ in Equation (1), then $T$ simultaneously transforms each of the vectors from one coordinate representation to the other:

$$
\mathrm{M}_{\mathrm{X}}=\mathrm{T}_{\mathrm{XX}} \cdot \mathrm{M}_{\mathrm{X}}
$$

Let us use the directions of two vectors $\vec{A}$ and $\vec{B}$, which are known in both $X$ and $X^{\prime}$ coordinates, to construct a set of three orthonormal vectors in each coordinate system. One of several possible sets is $\hat{A}, A \hat{x} B$, and $\hat{A} \times(A \hat{x} B)$. The circumflex denotes a vector normalized to unit length $\hat{A}=\frac{\vec{A}}{|A|}$ (equivalent to the direction cosines of the vector), and $A \hat{x} B$ denotes that the normalization must be done after the cross product operation is performed. The relationship of these vectors is indicated in Figure 1. Since the identity of the vectors is independent of the coordinate representation, Equation (2) will hold if we take

$$
M=[\hat{A}, A \hat{x} B, \hat{A} \times(A \hat{x} B)]
$$

on each side of the equation. Furthermore, M so defined is orthonormal, so $\mathrm{M}^{-1}=\mathrm{M}^{\mathrm{t}}$. We may immediately solve for $\mathrm{T}$ :

$$
\begin{aligned}
\mathrm{T}_{\mathrm{XX}} \mathrm{X}^{\prime} & =\mathrm{M}_{\mathrm{X}} \mathrm{M}_{\mathrm{X}^{\prime}}^{\mathrm{t}} \\
& =[\hat{\mathrm{A}}, \mathrm{A} \hat{\mathrm{x}} \mathrm{B}, \hat{\mathrm{A}} \mathrm{x}(\mathrm{A} \hat{\mathrm{x}} \mathrm{B})]_{\mathrm{X}} \quad[\hat{\mathrm{A}}, \mathrm{A} \hat{\mathrm{x}} \mathrm{B}, \hat{\mathrm{A}} \mathrm{x}(\mathrm{A} \hat{\mathrm{X}} \mathrm{B})]_{X^{\prime}}^{\mathrm{t}}
\end{aligned}
$$

Because $T$ can transform any vector between the $X$ and $X^{\prime}$ coordinate representations, T determines the relative "attitude" of the two coordinate systems. It is clear from Equation (3) that information about the magnitude of $\vec{A}$ or $\vec{B}$ is unnecessary, and also (see Figure 1) that 
information about the angle $\theta_{\mathrm{AB}}$ has been lost. In applications, therefore, because of the sense in which the cross product is taken, $A$ is chosen to correspond to the quantity whose direction is more accurately known, and $\vec{B}$ is used only to define the plane in which both vectors lie. Equation (3) always has a solution if $\vec{A}$ and $\vec{B}$ are not parallel or antiparallel. Obviously, the choice of coordinate systems $X$ and $X^{\prime}$ is completely arbitrary. Even in a dynamic system, Equation (3) holds at any instant. The time dependence of $T$ may be determined by inserting $\hat{A}(t)$ and $\hat{B}(t)$ in the equation.

Information can be extracted from $T$ concerning the single rotation about some appropriate axis that will rotate the $X^{\prime}$ system into conjunction with the $\mathrm{X}$ system. The direction cosines of the axis of rotation are the components of the eigenvector $\hat{E}$ of the eigenvalue equation

$$
(\mathrm{T}-[1]) \hat{\mathrm{E}}=0 \text {. }
$$

The rotation angle about the $\hat{E}$ direction is

$$
\Phi=\arccos \left\{\frac{1}{2}(\text { Trace } T-1)\right\} \text {. }
$$

The components of $\mathrm{T}$ express the directions of the coordinate axes $x_{1}^{\prime}, x_{2}^{\prime}, x_{3}^{\prime}$ of the $x^{\prime}$ system in the $x$ system. To illustrate this we take, as a special case, $\hat{A}, A \hat{x} B$, and $\hat{A} \times(A \hat{x} B)$ parallel to the axes of the $X^{\prime}$ coordinate system. Then $\mathrm{M}_{\mathrm{X}^{\prime}}=[1]$ and Equation (3) reduces to

$$
T_{X X}=[\hat{A}, A \hat{x} B, \hat{A} x(A \hat{x} B)]_{X}=\left[x_{1}^{\prime}, x_{2}^{\prime}, x_{3}^{\prime}\right]_{X} .
$$

This special case of the "double-cross" transformation was used by Russell (1971), who also appreciated the inconvenience of the Eulerian angle approach.

\section{Applications}

In a typical application for rockets or low altitude satellites, $\hat{A}$ and $\hat{B}$ might be identified with the directions of the solar and the geomagnetic field vectors, $X^{\prime}$ might be spacecraft coordinates, and $X$ might be geocentric equatorial inertial coordinates. 
Another choice for $\hat{A}$ or $\hat{B}$ might be the total angular momentum vector of a spinning spacecraft, since it is often known in both inertial and spacecraft coordinates. Definitions of, and transformations between common geophysical coordinate systems using the matrix transformation approach have been reported in the literature by Russe11 (1971), or may be found in a Goddard Space Flight Center document (1970).

Not uncommonly, it requires considerable computation to determine appropriate vectors in the spacecraft coordinate system. To provide a useful example, we shall consider the determination of the solar direction, denoted by $\hat{S}$, in spacecraft coordinates using three solar sensors. Two of these sensors have "look directions" $\hat{C}$ and $\hat{D}$, such that the angles $\theta_{C S}$ and $\theta_{D S}$ between the sensor look directions and the solar direction are measured. Thus $\hat{S}$ may be considered to lie at the intersection of two cones with vertices at the origin, with axes $\hat{C}$ and $\hat{D}$, and with half-angles $\theta_{C S}$ and $\theta_{D S}$, as depicted in Figure 2 . There are in general (for $\hat{C} \neq \hat{D}$ ) two solutions for $\hat{S}$, and further information provided by the third sensor, which must have a look direction not in the plane of $\hat{C}$ and $\hat{D}$, is necessary to resolve the ambiguity. (Note: For rockets or low altitude satellites, $\hat{C}$ (or $\hat{D}$ ) might, instead, be the geomagnetic field vector as determined by an on-board magnetometer. In this case, the angle $\left({ }{ }_{\mathrm{CS}}\right)$ between the geomagnetic and solar directions need not be measured. This angle is obviously independent of the coordinate system and can easily be calculated from ephemeris data). The problem of the intersection of two cones can most easily be solved in the coordinate system ( $X^{\prime \prime}$ ) of Figure 2. The direction cosines of the two solutions for $\hat{S}$ are: 


$$
\begin{aligned}
& \mathrm{s}_{3}=\cos \theta_{\mathrm{CS}} \\
& \mathrm{s}_{2}=\frac{\cos \theta_{\mathrm{DS}}-\cos \theta_{\mathrm{CD}} \cos \theta_{\mathrm{CS}}}{\sin \theta_{\mathrm{CD}}} \\
& \mathrm{s}_{1}= \pm \sqrt{1-\mathrm{s}_{2}{ }^{2}-\mathrm{s}_{3}^{2}}
\end{aligned}
$$

where $\theta_{C D}=\arccos (\hat{C} \cdot \hat{D})$, and the ambiguity in $S_{1}$ must be resolved with additional information. Equatiors (4) are inaccurate if $\hat{C}$ and $\hat{D}$ are nearly parallel or antiparallel. The cones do not intersect if $s_{1}$ nas no real root. To transform $\hat{S}$ from $X^{\prime}$ ' coordinates to spacecraft ( $X^{\prime}$ ) coordinates we use the special case of a "double-cross" transformation:

$$
\begin{aligned}
& \qquad M_{X^{\prime}}=[D \hat{x} C, \hat{C} x(D \hat{x} C), \hat{C}]_{X^{\prime}} \\
& \qquad M_{X^{\prime}},=[1]=M_{X^{\prime}}^{t} \\
& \hat{S}_{X^{\prime}}=T_{X^{\prime} X^{\prime}}, \hat{S}_{X^{\prime}}=M_{X^{\prime}} M_{X^{\prime}} t^{\prime} \hat{S}_{X^{\prime}},=M_{X^{\prime}} \hat{S}_{X^{\prime}}, \cdot \\
& \text { At this point } \hat{S}_{X^{\prime}} \text { may be identified with } \hat{A} \text { or } \hat{B} \text { and the attitude matrix } \\
& T_{X X} \text { found as described previously. }
\end{aligned}
$$




\section{Acknowledgements}

This work was performed while the author was a National Academy of Sciences Research Associate in the Magnetic and Electric Fields Branch at NASA-Goddard Space Flight Center, Greenbelt, Maryland 20771. The helpful comments of Dr. B. G. Ledley are gratefully acknowledged. 
REFERENCES

Goldstein, H., Classical Mechanics, Addison-Wesley, Reading, Mass., 1950.

Greensite, A. L., Analysis and Design of Space Vehicle Flight Control

Systems, Spartan Books, New York, 1970.

Magnetic and Electric Fields Branch, Coordinate transformations used in OGO Satellite data analysis, NASA-Goddard Space Flight Center Document X-645-70-29, January, 1970.

Marion, J. B., Classical Dynamics, Academic Press, New York, 1965.

Russe11, C. T., Geophysical Coordinate Transformations, Cosmic Electrodynamics, $2,184-196,1971$.

Singer, S. F., (ed.), Torques and Attitude Sensing in Earth Satellites, Academic Press, New York, 1964.

Thomson, W. T., Introduction to Space Dynamics, John Wiley and Sons, New York, 1961. 


\section{FIGURE CAPTIONS}

Figure 1: Three orthonormal vectors $\hat{A}, A \hat{x} B$, and $\hat{A} \times(A \hat{x} B)$ created from two known vectors $\vec{A}$ and $\vec{B}$. Information about the angle $\theta_{A B}$ is lost, and knowledge of the magnitudes of $\vec{A}$ or $\vec{B}$ is unnecessary.

Figure 2: Natural coordinate system for finding the intersection of two cones having the same vertex. The two vectors $\hat{S}^{+}$and $\hat{S}^{-}$are the solutions sought. Information in addition to $\hat{\mathrm{D}}, \hat{\mathrm{C}}, \theta_{\mathrm{DS}}$ and $\theta_{\mathrm{CS}}$ must be provided to distinguish between $\hat{S}^{+}$and $\hat{S}^{-}$. 


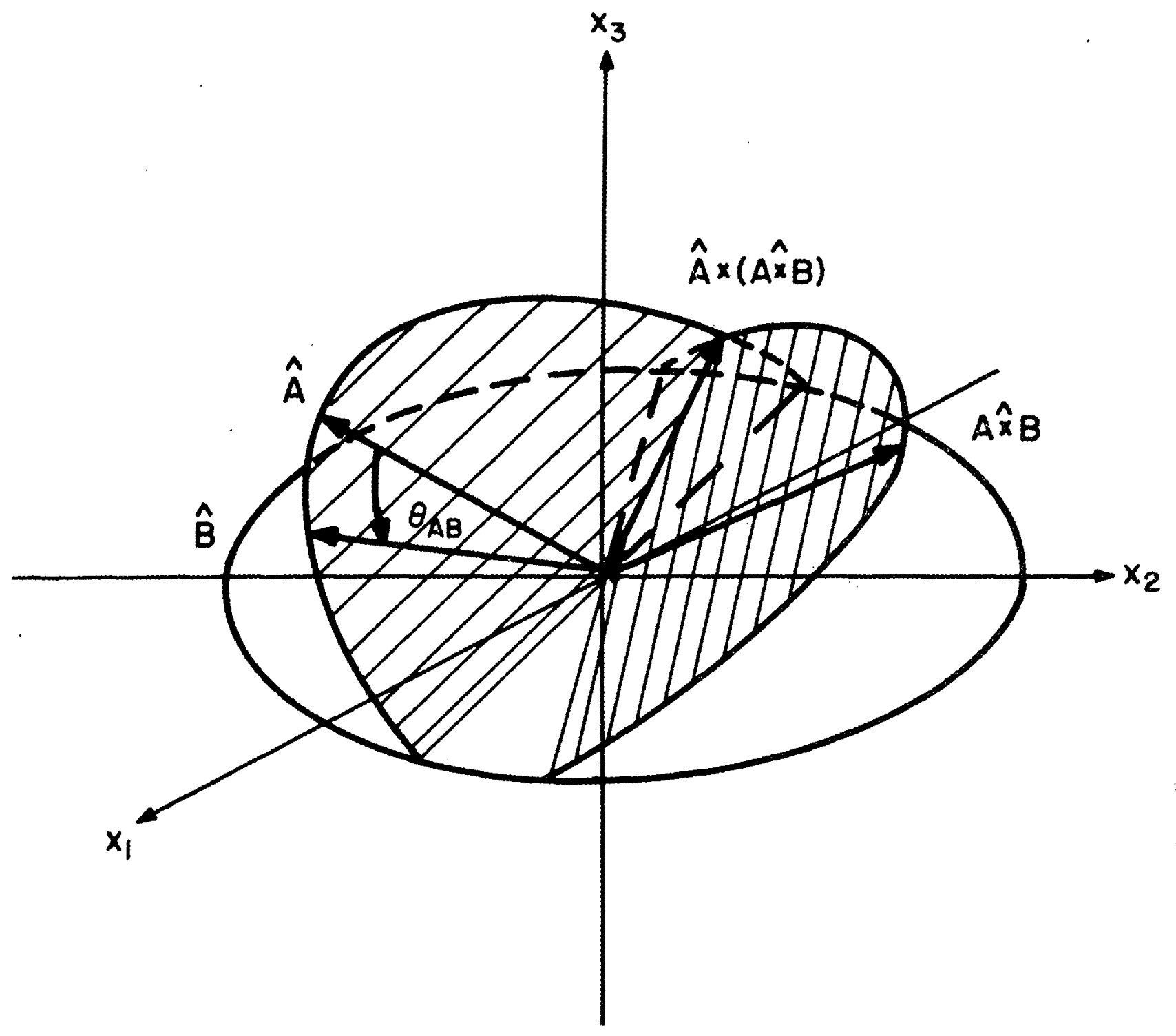

FIGURE 1 


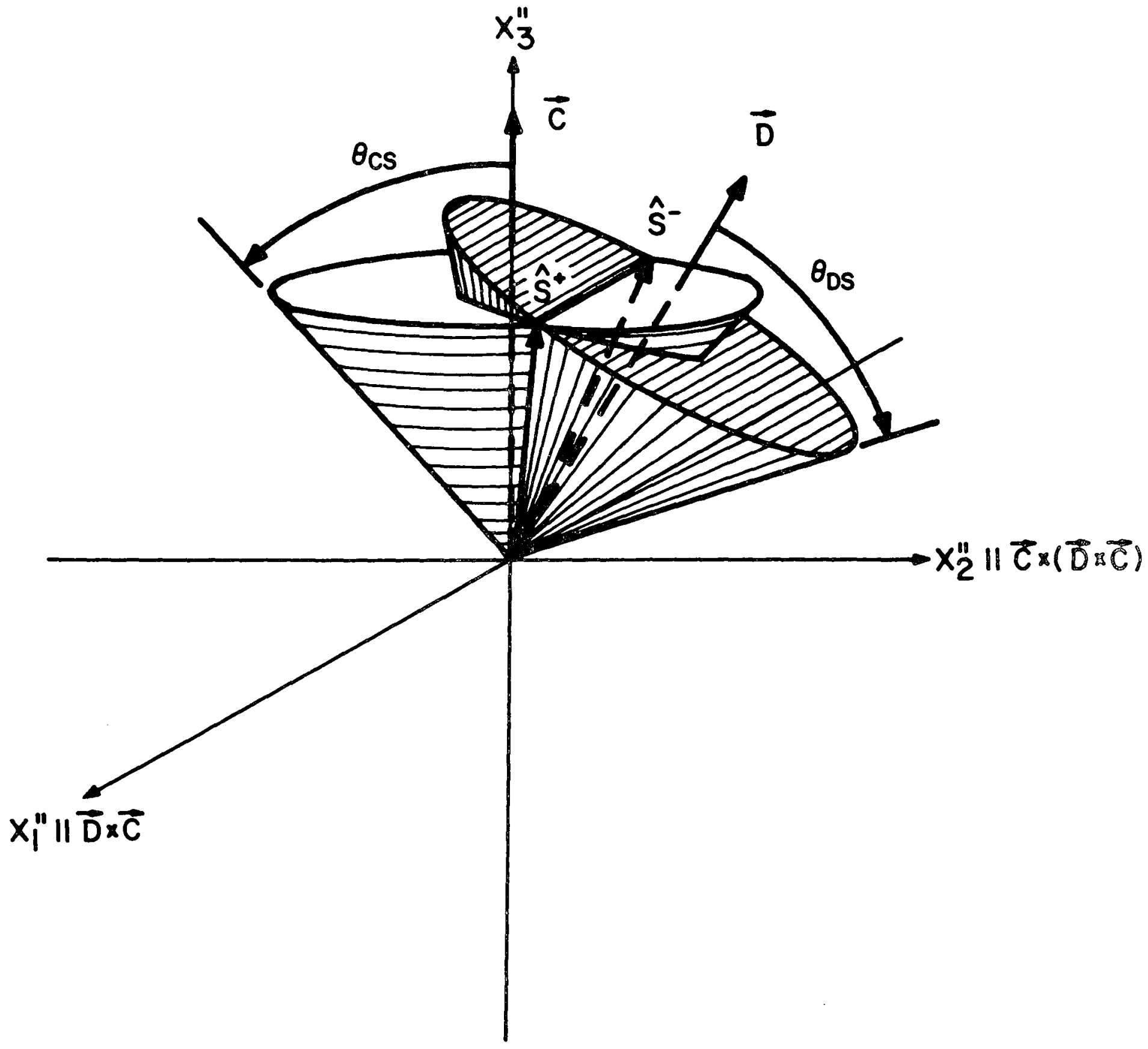

FIGURE 2 